\title{
A System to Distribute Navy Coastal Ocean Model Data Using the Open Geospatial Consortium's Web Map Service Protocol
}

\author{
Norman C. Schoenhardt, Elias Z. Ioup, Frank P. McCreedy \\ Naval Research Laboratory \\ Stennis Space Center, MS 39529
}

\begin{abstract}
A method for disseminating Navy Ocean Coastal Model (NCOM) data as imagery over the Internet via the Web Map Service (WMS) protocol is presented. NCOM data are provided in multidimensional numerical data files. Distribution of NCOM data via the WMS protocol requires the data be rendered into an image. The multidimensional properties of the NCOM data allow the plotted data images to be grouped into layers based on dimensions. The NCOM WMS supports optional functionalities provided by the WMS protocol, allowing users to obtain detailed information about map images created by the NCOM WMS. By serving NCOM data via the WMS protocol, clients can access the data using common geographic information system (GIS) client software.
\end{abstract}

\section{INTRODUCTION}

The Navy Coastal Ocean Model (NCOM) was developed to meet the Navy's needs for coastal ocean simulation and prediction. NCOM runs produce multidimensional acoustic and environmental ocean condition data for present time and predictions for future ocean conditions. NCOM data are used in a variety of activities such as search and rescue, storm surge prediction, mission analysis, and a wide array of other Navy applications [1]. A Web Map Service (WMS) produces maps or images of spatially referenced data dynamically from geographic information [2]. Clients can access the WMS service operations using a standard Web browser by submitting requests in the form of Universal Resource Locators (URLs) or through the use of common geographic information system (GIS) client software that supports the WMS protocol [2].

A WMS server application was developed to serve NCOM data as data plot images over the Internet. The NCOM WMS server implements the WMS protocol's mandatory operations as well as one optional one. Two main components comprise the WMS server for NCOM data. The first is a layering structure the WMS server publishes to provide a list of every map image the server will provide. The second is a method for rendering NCOM data into imagery.

Additionally, the NCOM WMS server was developed to support the optional WMS capability known as the GetFeatureInfo request. The GetFeatureInfo request allows users to query the WMS to obtain model data values and metadata for a specific geographic points.

\section{LAYERING SCHEME}

In the WMS protocol the geographic information content offered for portrayal by a WMS server is organized into layers [2]. Each layer represents one distinct map provided by the service. The WMS server publicizes all available layers in a WMS capabilities document. The capabilities document is an XML document that contains information and metadata for mandatory and optional content of the service. Clients can access the capabilities document by sending a GetCapabilities request to the WMS Server. Once the capabilities document is received the client has access to every available layer the WMS server provides and can use the layer names to make data requests. Therefore, when creating a WMS server it is necessary to develop a layering scheme for the geographic content the server will provide.

To better understand the layering system used in the WMS service presented here, it is necessary to first discuss the structure of the NCOM-generated data. NCOM data are produced in two distinct resolution sets; a global dataset and a regional dataset. The global dataset is comprised of separate geographic regions which when combined provide complete worldwide coverage. It is produced at a 1/8-degree resolution and can have 42 vertical layers depending on the data product type [3]. The global dataset has a 72-hour forecast period separated into 3 hour increments known as tau values. The tau values act as offsets to the time in which the model was run, also known as the base reference time, and increment by a value of three. Data are produced for each tau increment until 72 hours are reached. Therefore a tau value of 0 would represent data produced at the base reference time, a tau value of 3 would represent ocean conditions three hours past the base reference time and so on. The regional NCOM data 
comprise a higher resolution dataset designed to provide more accurate detail of an area. As the name suggests, the regional data is produced for specific geographical regions around the world and does not provide complete worldwide coverage. The resolution of this dataset can vary but is roughly $1 / 36$ of a degree. Regional NCOM data have as many as 41 vertical layers and a 72-hour forecast period.

NCOM produces a number of products that are identical for both resolution data types. These products, also known as parameters, model different oceanic conditions or acoustic properties. Six NCOM standard parameters are supported by the NCOM WMS system: salinity, sea surface height, temperature, sound speed, Eastward water velocity, and Northward water velocity. The WMS additionally supports fifteen RP33 parameters: acoustic deep sound channel-axis depth, acoustic deep sound channel-axis depth excess, acoustic half-channel mask, acoustic surface duct mask, acoustic surface duct-sonic layer depth, acoustic surface duct-cutoff frequency, acoustic surface duct-mixed layer depth physical oceanography, acoustic secondary sound channel-one axis depth, acoustic secondary sound channel-one cutoff frequency, acoustic conjugate depth-1 depth excess, acoustic conjugate depth-2 depth excess, acoustic conjugate depth-3 depth excess, acoustic conjugate depth-4 depth excess, acoustic conjugate depth-5 depth excess, and acoustic conjugate depth-6 depth excess.

When developing a layering scheme, it must not only contain all possible layers provided by the WMS service, but also allow for ease of navigation. Due to the complexity of the NCOM data, a tree structure approach was adopted. In this structure, each branch in the tree would represent a dimension in the NCOM data. The first level of layers or branches is the resolution data type. This level divides the NCOM data into two layer groups; global and regional. Next, layers are grouped by geographical regions. This subdivides the data further and allows users to find NCOM data based on geographical areas of interest. An additional branch was added for parameter grouping. Parameters with similar characteristics were organized into groups, representing the next subdivision of data. The layers are then partitioned by parameter type, followed by forecast time or tau value and finally, if the data type supports it, by depth. By structuring the NCOM layers in this fashion, users can quickly find data based on resolution, region, parameter, forecast time and elevation.

The layer names are constructed using the name of each dimension to which the data belong. This naming convention serves two purposes. The first is to provide the user with information of what type of data the layer contains. The second is to allow the NCOM WMS server to parse the layer name and quickly locate and extract the data based on the dimensions provided in the layer name. Figure 1 depicts a partial view of the NCOM layer tree structure.

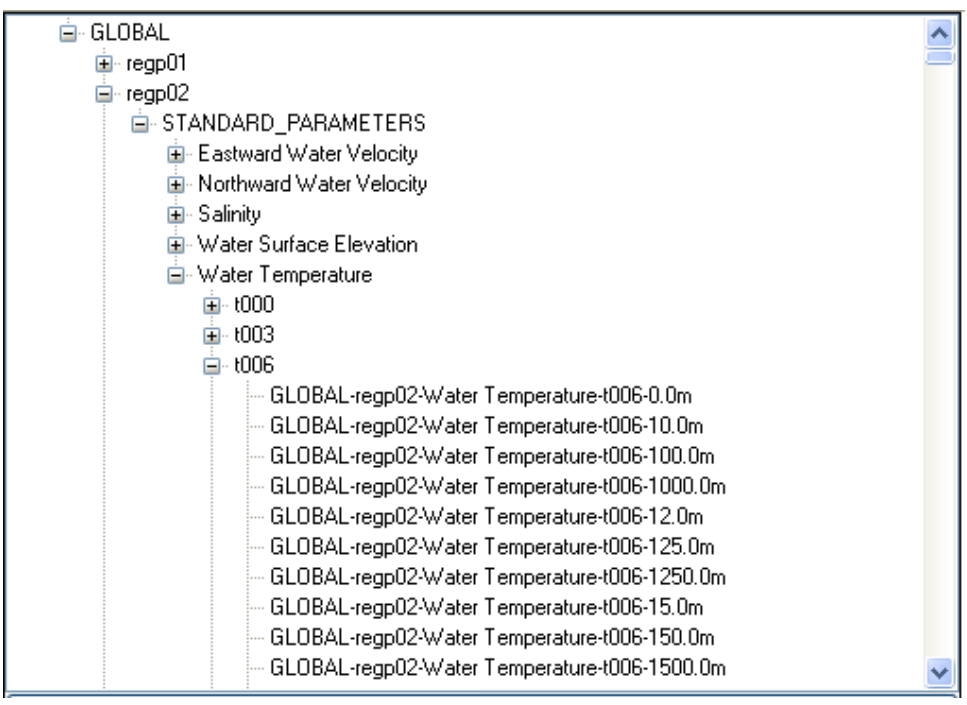

Figure 1 - Example of global NCOM layer tree structure

When using the layer structure defined above with datasets that contain multiple facets, two problems arise. Since NCOM data contain multiple dimensions broken into several geographic regions the layer structure required to represent each individual layer becomes extremely large. As stated above, the WMS protocol allows for a capabilities document to return all available layers the WMS service will provide. The larger the layer structure becomes, the larger in size the capabilities document must be to accommodate every possible layer. Since the capabilities document is sent over a network to a requesting client, the size of the document determines the speed in which the document is received. In instances of low bandwidth connections large capabilities documents can significantly reduce the performance of the WMS. Secondly, to display NCOM data in a worldwide view using 
the layering scheme above, multiple layers must be active or viewable at a single time. The problem with this is many GIS clients allow only a small number of layers to be active at any given time. Therefore, displaying data from multiple parameters over different time and elevation dimensions for the entire world becomes extremely difficult, or even impossible. To combat these issues a special layer known as an automatic layer, or auto layer, was developed.

The auto layer is a single layer of worldwide coverage composed of like data from multiple geographic regions. Auto layers are composed of datasets of the same parameter type, forecast time, and depth value. The combined datasets define the total geographical area that are to be covered by a particular auto layer. When a request is received for an auto layer, the WMS server will collect the NCOM datasets matching the requested parameter type, forecast time, and depth value; merge them together; and produce the final image.

The NCOM WMS Service offers auto layers for global NCOM datasets and regional NCOM datasets. Additionally, it offers a set of auto layers that merge both global and regional datasets to produce a single set of auto layers for all NCOM data. In order to merge the different resolution datasets the NCOM WMS server application incorporates a set of predefined business rules. The business rules are a strict set of guidelines used to determine which of two overlapping data sets takes precedence. The business rules guarantee the highest resolution or highest quality data always takes precedence providing imagery that shows the highest quality data available, back filled with subsequent lower resolution or lower quality data. Figure 2 and Figure 3 show an NCOM temperature auto layer created by merging all regional temperature data with all global temperature data.

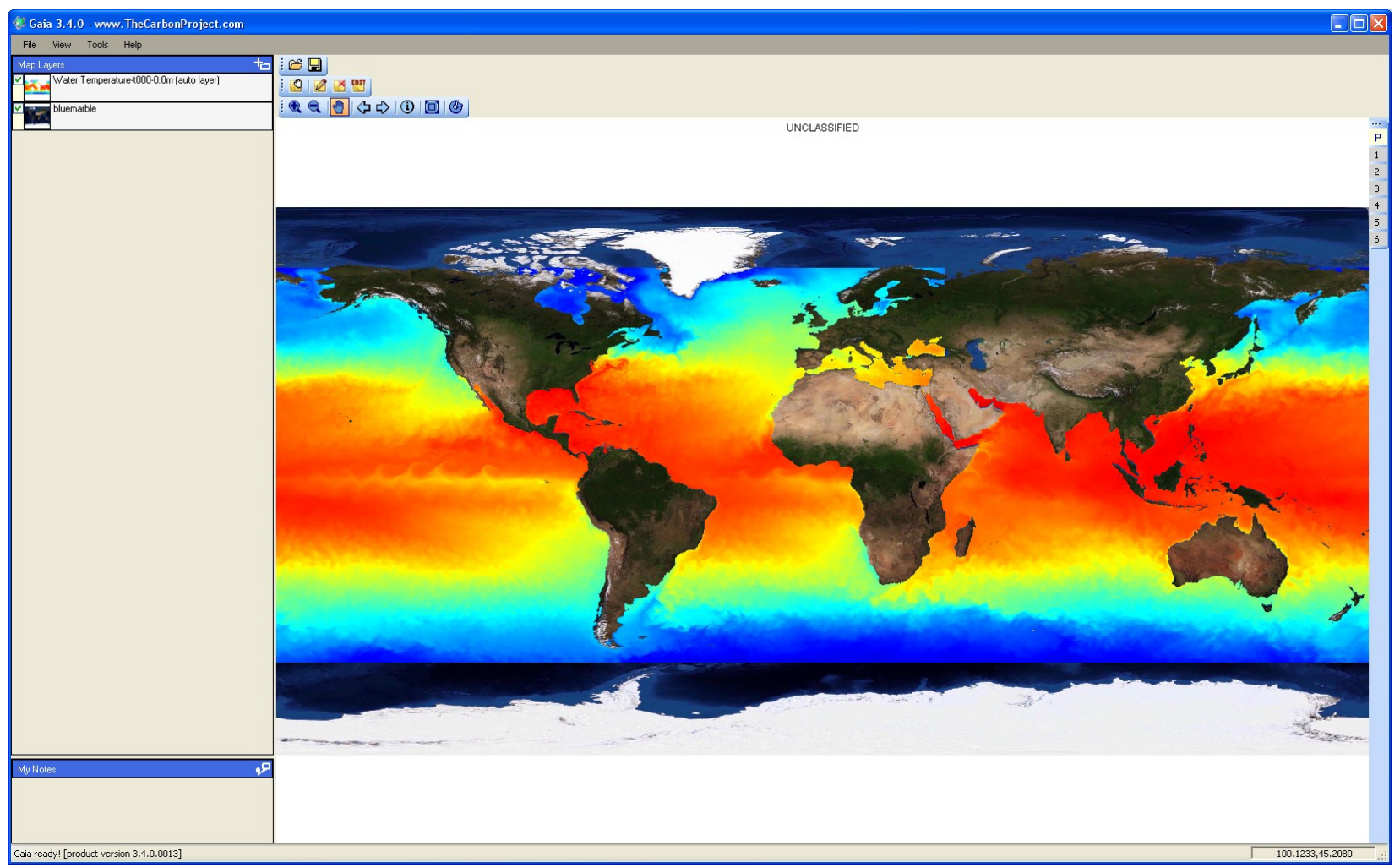

Figure 2 - Example of NCOM auto temperature layer (regional data merged with global data) with NASA Blue Marble background 


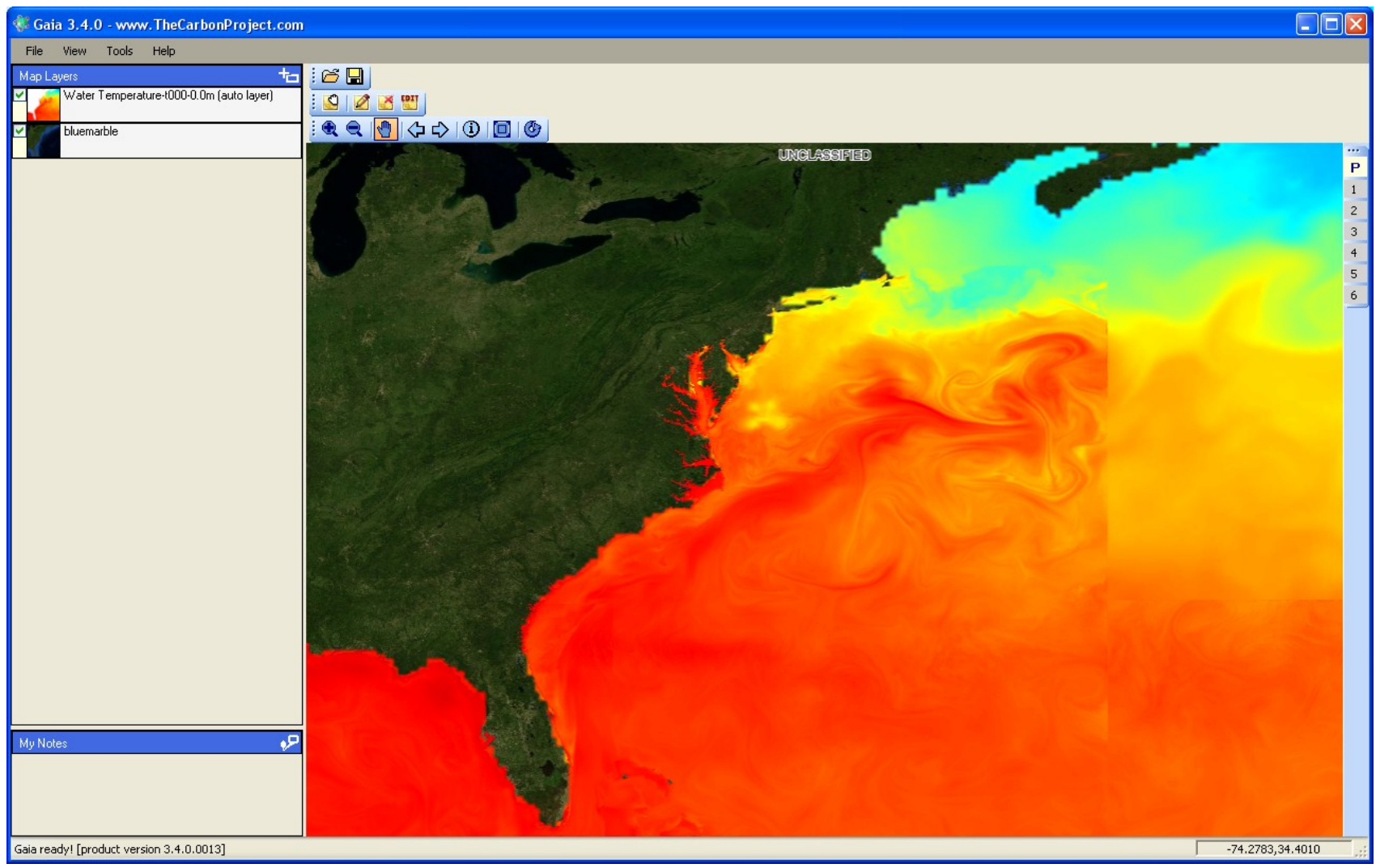

Figure 3 - Closer view of NCOM auto temperature layer (regional data merged with global data)

The NCOM WMS server also supplies a layer depicting current vectors to complement the NCOM imagery. The current vectors are created using the NCOM Eastward and Northward water velocity parameters (current U and current V components). Directional arrows are created by computing velocity and direction. Once the direction and velocity have been calculated, the arrows are drawn into a transparent image to produce the current vector layer. Figure 4 shows an NCOM layer with the ocean current layer overlaid.

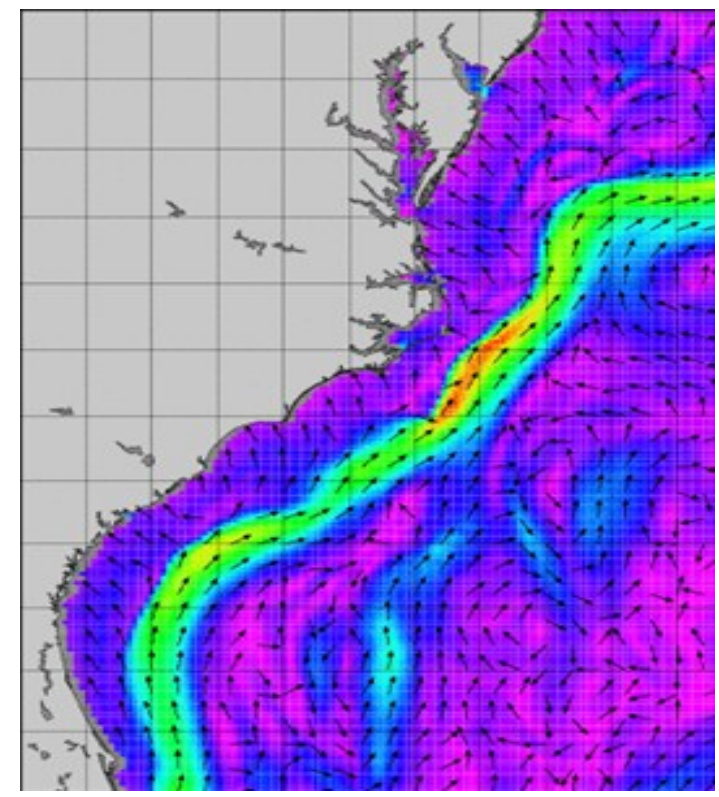

Figure 4 - NCOM sea surface height with current vector layer overlaid 


\section{IMAGE CREATION}

A WMS provides geographic information as imagery through the use of a GetMap request. A client can submit a GetMap request via a URL which supplies the WMS server with information needed to generate the imagery [4]. The request contains information such as the layer name(s) requested, geographic area of interest, desired coordinate reference system (CRS), height, width, and format of the image to be returned and other required and optional information. The WMS server then uses this information to transform the geographic data into the image requested.

NCOM data are provided as multidimensional numerical grids in the Network Common Data Form (NetCDF) format. NetCDF is open-source software that supports the creation, access, and sharing of scientific data and metadata using machine-independent data formats. NetCDF provides flexibility and efficiency of data access and has become a standard for model outputs in the ocean modeling communities [5]. The NCOM parameter data stored in NetCDF files are either three- or four-dimensional. The dimensions are latitude, longitude, forecast time, and depth. The latter is only present in the four-dimensional datasets. Each NCOM NetCDF file stores data values for one or more parameters and metadata associated with the parameters.

The multidimensional NCOM grids must be scaled down to a two-dimensional array of values in order to render them into images. Dimensionality reduction is accomplished by slicing the single multidimensional grid into multiple two-dimensional grids for each forecast time and depth (if applicable). The two-dimensional grid is rendered into an image for each parameter value in the grid. Thus, a single NCOM grid becomes (forecast times $\mathrm{x}$ depths $\mathrm{x}$ parameters) images. The image size is the same as the size of the grid slice; each grid point becomes a pixel in the image. The color of the pixels is determined by the parameter type represented in the image. Each parameter type generated by NCOM has an associated color table that contains a fixed number of color values along with the data ranges to which each color is assigned. The individual data values are matched to a color based on the associated color map and the pixel that represents the data value is set to that color.

As stated above, the WMS protocol allows clients to specify a geographical area of interest as well as the image dimensions to visualize that area. In most instances the resolution calculated from the supplied area and image size will not be the same as the data's underlying resolution. Since the image is created using the data's underlying resolution to maintain the one-to-one data value to pixel ratio, the image must be scaled to fulfill the client's request. To do this, a scaling method that uses bilinear interpolation to scale the image to the requested size was developed.

\section{DetAILED MODEL INFORMATION}

The WMS protocol provides optional functionality for a client to receive more detailed information about the underlying data of requested map images [2]. This information is made available through the GetFeatureInfo request. The canonical use case for the GetFeatureInfo request is that a user sees the response of a map request and chooses a point (I,J) on that map for which to obtain more information. The basic operation provides the ability for a client to specify which pixel is being queried, which layer(s) should be investigated, and what format the information should be returned in [2].

The NCOM WMS server provides support for the GetFeatureInfo request. Clients to the NCOM WMS server submit GetFeatureInfo requests and in return receive detailed information about the layer(s) requested at the given point. This information includes the latitude and longitude of the pixel point given; the underlying data value for the layer requested; the units of measure the data value is given in; the forecast time; depth if applicable; the classification level of the data; and all other parameter metadata of the type represented by the requested layer. The NCOM WMS server can return the information in one of two formats: an HTML format to allow for human readability or an XML format for not only human readability but also for use in other software applications. Figure 5 depicts and excerpt from a GetFeatureInfo response from the NCOM WMS server. 


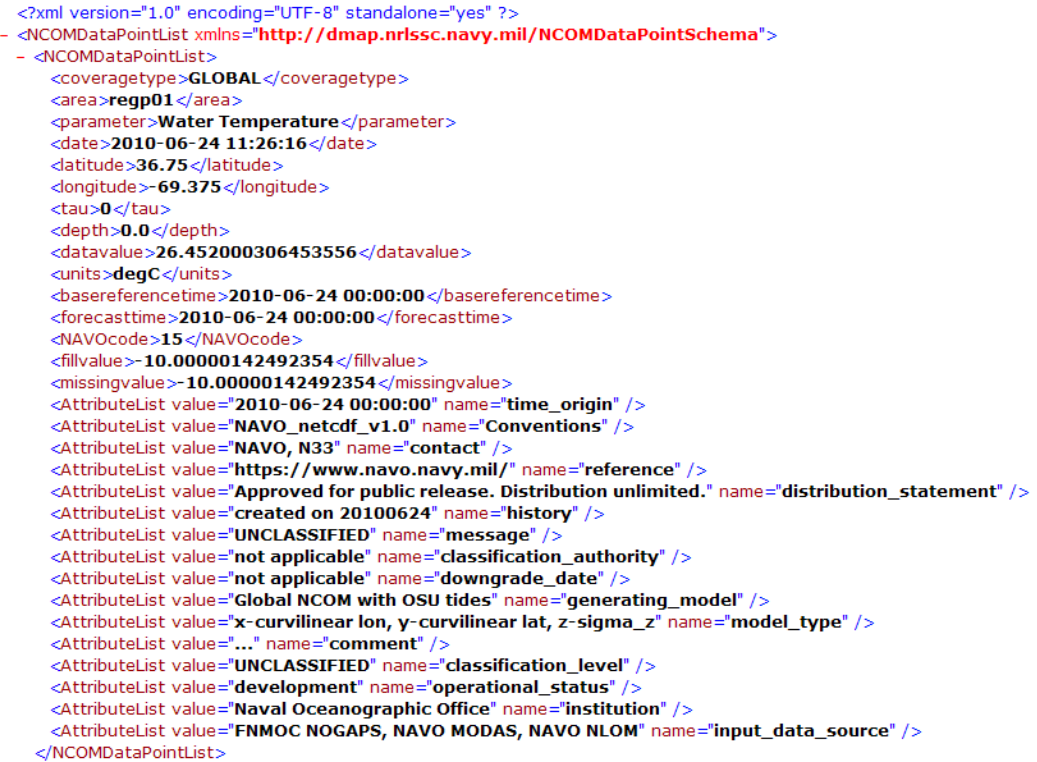

Figure 5 - Excerpt from NCOM WMS server XML formatted GetFeatureInfo response

\section{SUMMARY}

The Open Geospatial Consortium's Web Map Service protocol provides a vehicle to serve geographic information as imagery over the Internet. The NCOM WMS developed utilizes the WMS protocol to provide NCOM data as rendered images. By accessing this service, clients have access to a wide array of NCOM products. Through the use of a custom-developed layer structure, users can quickly locate the desired NCOM data. The addition of the auto layers also provides clients access to a broader range of NCOM products in a worldwide spectrum. The NCOM WMS server provides access to detailed information for the imagery it provides through the WMS protocol's GetFeatureInfo request.

\section{ACKNOWLEDGMENT}

This work was sponsored by the U.S. Naval Research Laboratory’s Base Program, Program Element No. 0602435N.

\section{REFERENCES}

[1] "First Global Model Developed at NRL Stennis." United States Naval Research Laboratory. Naval Reserch Laboratory, 30 Oct. 2006. Web. 27 July 2010. http://www.nrl.navy.mil/pao/pressRelease.php?Y=2006\&R=55-06r.

[2] Open Geospatial Consortium Inc. “OpenGIS ${ }^{\circledR}$ Web Map Server Implementation Specification.” (version 1.3.1) http://portal.opengeospatial.org/files/?artifact id=14416. (March 15, 2006).

[3] C.N. Barron, A.B. Kara, P.J. Martin, R.C. Rhodes, L.F. Smedstad (2006) "Formulation, implementation and examination of vertical coordinate choices in the Global Navy Coastal Ocean Model (NCOM)" Ocean Modeling, 11 (3-4), pp. 347-375.

[4] J. Sample, R. Ladner, L. Shulman, E. Ioup, et. al. "Enhancing the US Navy's GIDB Portal with Web Services." IEEE Internet Computing, Vol. 10, Num. 5 2006.

[5] S. Hankin, et. al. "NetCDF-CF-OPeNDAP: Standards for Ocean Data Interoperability and Object Lessons for Community Data Standards Processes." in Proc. OceanObs '09: Sustained Ocean Observations and Information for Society, Vol. 2, Venice, Italy, 21-25 September 2009. 
